\title{
Interférences
}

Ars scribendi

11 | 2018

Poésie latine et miroir du prince

\section{Au-delà du casque à cornes : Théodoric, monarque parfait et ami des poètes}

\section{Camille Bonnan-Garçon}

\section{(2) OpenEdition \\ 1 Journals}

Édition électronique

URL : http://journals.openedition.org/interferences/6531

DOI : 10.4000/interferences.6531

ISSN : $1777-5485$

Éditeur

HiSoMA - Histoire et sources des Mondes antiques

\section{Référence électronique}

Camille Bonnan-Garçon, « Au-delà du casque à cornes : Théodoric, monarque parfait et ami des poètes », Interférences [En ligne], 11 | 2018, mis en ligne le 26 juillet 2018, consulté le 15 septembre 2020. URL : http://journals.openedition.org/interferences/6531; DOI : https://doi.org/10.4000/ interferences.6531

Ce document a été généré automatiquement le 15 septembre 2020.

Tous droits réservés 


\title{
Au-delà du casque à cornes : Théodoric, monarque parfait et ami des poètes
}

\author{
Camille Bonnan-Garçon
}

1 Dans l'imaginaire populaire, le Romain voit le Barbare comme un être dépourvu de raffinement. Sidoine Apollinaire quant à lui n'était peut-être pas de cet avis. En effet, il commence ainsi la deuxième lettre du recueil de sa correspondance (Sidon., Ep. I, $1^{1}$ ) :

Saepe numero postulauisti ut, quia Theodorici regis Gothorum commendat populis fama ciuilitatem, litteris tibi formae suae quantitas, uitae qualitas significaretur

"À de nombreuses reprises, tu m'as prié dans tes lettres de te faire connaître l'extérieur et les habitudes de Théodoric, roi des Goths, dont la renommée populaire vante la politesse. »

2 Sidoine Apollinaire (430-486), poète et épistolier, fut le panégyriste officiel de trois empereurs. Il était également le gendre du premier d'entre eux, Avitus. Après la mort de celui-ci, en 456, il s'attache à son successeur Majorien, et prononce son panégyrique en 458. Enfin, après une retraite de sept ans en Auvergne, après l'assassinat de Majorien en 461, il compose en 468 le panégyrique d'Anthémius. Il fut également préfet du prétoire des Gaules et préfet de Rome. Sidoine est donc un homme proche du pouvoir, du pouvoir romain de la fin de l'Empire, mais aussi du pouvoir des rois barbares, puisqu'il passe notamment sept ans auprès de la cour du roi Théodoric II, à Toulouse. Sidoine se situe à la charnière entre la fin du régime impérial et le régime royal wisigothique. Marc Reydellet le décrit d'ailleurs comme un «homme de confluences ${ }^{2}$ ».

3 Cet attachement au pouvoir est visible dans sa poésie, notamment à travers ses Panégyriques, mais ce n'est pas ce volet de sa littérature encomiastique que nous allons prioritairement étudier, mais surtout la représentation qui transparaît chez Sidoine, non pas d'un empereur, mais bien d'un roi barbare, le roi des Wisigoths Théodoric II.

4 En littérature latine, aussi bien dans la poésie triomphale que dans l'historiographie, la description des chefs barbares se caractérise par l'emphase et la caricature, tant dans une vocation méliorative ${ }^{3}$ que péjorative, quand elle se caractérise par sa démesure et 
sa perfidie ${ }^{4}$. Ammien Marcellin, à la même époque que Sidoine, décrit par exemple de façon particulièrement empathique, caricaturale et standardisée le roi vandale Chnodomaire $^{5}$ (Amm., 16, 12, 4) :

Agitabat autem miscebatque omnia sine modo ubique sese diffunditans et princeps audendi periculosa rex Chonodomarius.

«Mais le roi Chnodomaire agitait tout, faisant sentir sa présence partout et sans

limites, chef dans une dangereuse entreprise.»

5 On verra que ce n'est pas le cas chez Sidoine, où le roi barbare est décrit d'après observation, de façon très détaillée et subtile, quoique également assez emphatique. La chose est d'ailleurs d'autant plus étonnante que Sidoine a souvent été décrit comme un "barbarophobe", notamment par Edward Gibbon ${ }^{6}$. Sidoine n'est en effet pas tendre lorsqu'il décrit les Burgondes qui occupent ses terres, au début du carmen XII (Sidon., carm. 12, 1-22) :

Quid me, etsi ualeam, parare carmen

Fescenninicolo iubes Diones,

Inter crinigeras situm cateruas,

Et Germanica uerba sustinentem,

Laudantem tetrico subinde uultu,

Quod Burgundio cantat esculentus,

Infundens acido comam butyro?

Vis dicam tibi quid poma frangat?

Ex hoc barbaricis abacta plectris

Spernit senipedem stylum Thalia,

Ex quo septipedes uidet patronos.

Felices oculos tuos et aures,

Felicemque libet uocare nasum,

Cui non allia, sordidoeque ccepce,

Ructant mane nouo decem apparatus.

Quem non ut uetulum patris parentem,

Nutricisque uirum, die nec orto,

Tot tantique petunt simul gigantes,

Quod uix Alcinoi culina ferret.

"Qui ? Moi ? Même si je le pouvais, tu me demandes d'écrire des poèmes fescennins de Vénus alors que je suis parmi les hordes chevelues, que je suis forcé d'entendre le langage barbare du Germain et d'applaudir, en me faisant violence, à ce que chante le Burgonde ivre qui se parfume la tête d'un beurre rance? Veux-tu que je te dise pourquoi ma veine poétique se glace? Effrayée par la lyre discordante des Barbares, Thalie dédaigne des vers qui ont six pieds, depuis qu'elle voit des protecteurs qui en ont sept. Heureux tes yeux, tes oreilles, heureux ton nez luimême, car il ne sent pas dix fois chaque matin l'odeur fétide et de l'ail et de l'oignon! Tu n'es pas forcé, comme si tu étais leur grand-père, ou le mari de leur nourrice, de recevoir, avant le jour, ces géants que pourrait contenir à peine la cuisine d'Alcinoüs. »

6 Pour étudier cette représentation du roi barbare chez Sidoine, nous nous appuierons sur un corpus composé à la fois de lettres et de pièces en vers. La lettre I, 2 est entièrement consacrée à la description de Théodoric, qui, selon une structure très classique, fait suivre une description physique et morale générale du souverain du récit de l'une de ses journées, témoin de ses activités quotidiennes. Nous nous pencherons également sur le carmen 7, qui décrit in uiuo le roi Théodoric.

Chez Sidoine, on peut tout d'abord constater, d'après la lettre I, 2, que Théodoric est décrit comme un archétype du monarque parfait amateur de poésie et faisant preuve de la plus grande ciuilitas. Nous rapprocherons à cet effet deux scènes de banquet, 
extraites des lettres I, 2 (banquet chez Théodoric) et I, 11 (banquet chez l'empereur Majorien), ce qui nous permettra de voir que Sidoine construit en miroir les portraits de Théodoric et de Majorien. Cette réflexion nous amènera dans un second temps à comparer la description faite par Sidoine des trois empereurs dans leur panégyrique avec le roi Théodoric, afin de former par touche le portrait de l'empereur parfait.

\section{Théodoric, monarque parfait et protecteur des poètes}

8 La lettre I, 2 est entièrement consacrée à un portrait du roi wisigoth Théodoric. Sa place dans le recueil de la correspondance de Sidoine Apollinaire révèle assez l'importance de cette description. Elle trace le portrait complet du monarque, en glissant du raffinement de son physique au celui de son goût littéraire et mondain.

Le terme qui semble sous-tendre toute la description de Théodoric, et qui apparaît dès le premier paragraphe, est celui de ciuilitas. Si Marc Reydellet souligne le caractère politique du terme, étymologiquement lié à ciuis et exprimant des qualités liées à la façon de gouverner ${ }^{7}$, on ne peut en ignorer la première dimension mondaine. Ciuilitas

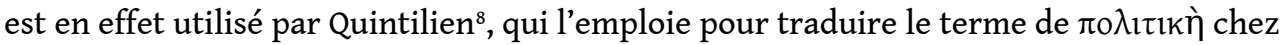
Aristote. Cependant, à partir de Suétone, ce terme prend un sens social et mondain. Il est notamment utilisé dans la Vie d'Auguste pour décrire l'attitude clémente de l'empereur envers des libellistes.

\section{Du raffinement physique au raffinement littéraire}

10 La description physique de Théodoric, tout en donnant parfois dans l'emphase, ne présente pas le caractère monstrueux des portraits de rois barbares habituels. La vigueur du roi wisigoth n'a d'égal que son raffinement presque romain. S'il se manifeste dans les pratiques vestimentaires du souverain, ce raffinement s'étend au style de la description de Sidoine. Théodoric en effet, contrairement aux Burgondes malpropres, ne néglige pas les soins du corps (Sidon., Ep. I, 2, 2) :

Pilis infra narium antra fruticantibus quotidiana succisio. Barba concauis hirta temporibus, quam in subdita uultus parte surgentem stirpitus tonsor assiduus genas ad usque forpicibus euellit.

"Chaque jour on lui coupe le poil qui pousse à l'ouverture des narines. Dans le creux de ses tempes, se hérisse une barbe touffue, et tous les jours un barbier assidu lui arrache à la pince à épiler celle qui pousse depuis le bas du visage jusqu'aux joues. »

11 Mais au-delà d'un raffinement presque romain, Théodoric est représenté avec un luxe de métaphores et de comparaisons, notamment dans la description de son visage, qui met en œuvre des métaphores raffinées rappelant la poésie amoureuse (Sidon., Ep. I, $2,3)^{9}$.

Aurium legulae, sicut mos gentis est, crinium superiacentium flagellis operiuntur. Nasus uenustissime incuruus. Labra subtilia, nec dilatatis oris angulis ampliata. Si casu dentium series ordinata promineat, niueum protinus repraesentat colorem.

"Ses oreilles, suivant la coutume de sa nation, sont couvertes par des cheveux qui descendent en tresses. Son nez est très agréablement arqué. Ses lèvres, minces, sont semblables à sa bouche dont les angles sont peu dilatés. Si, par hasard, ses dents se montrent avec leur gracieux alignement, elles offrent une blancheur de neige. » 
Ce raffinement physique n'est que le reflet d'un raffinement de conduite. La journée de Théodoric, racontée in extenso dans la lettre I, 2, se caractérise toujours par la ciuilitas politique et sociale, avec mesure et modération. Cette modération est également présente dans sa pratique du banquet. Théodoric en effet, en bon émule des monarques hellénistiques, pratique le symposion artistique et littéraire. Le repas a lieu traditionnellement le soir. La nourriture servie et le décor ne sont pas détaillés, d'abord parce qu'ils l'ont déjà été lors de la description du repas du midi, mais aussi parce que les spectacles donnés durant le banquet sont davantage mis en valeur que le repas, ce qui est courant dans les descriptions de banquet (Sidon., Ep. I, 2) :

Circa nonam recrudescit moles illa regnandi. Redeunt pulsantes, redeunt submouentes, ubique litigiosus fremit ambitus: qui tractus in uesperam, coena regia interpellante rarescit, et per aulicos deinceps pro patronorum uarietate dispergitur, usque ad tempus concubiae noctis excubaturus. Sane intromittuntur, quanquam raro, inter coenandum mimici sales, ita ut nullus conuiua mordacis linguae felle feriatur. Sic tamen quod illic nec organa hydraulica sonant, nec sub phonasco uocalium concentus meditatum acroama simul intonat. Nullus ibi lyristes, choraules, mesochorus, tympanistria, psaltria canit : rege solum illis fidibus delinito, quibus non minus mulcet uirtus animum quam cantus auditum.

«Vers la neuvième heure, la charge du trône refait surface. Viennent les solliciteurs, viennent ceux qui les éloignent; partout frémissent la cabale et l'intrigue. La foule s'éclaircit à l'approche du souper du roi, puis se disperse chez les courtisans, chacun auprès de son patron, jusqu'au milieu de la nuit. Quelquefois, mais rarement, on donne pendant le souper un libre cours aux saillies des mimes, de manière toutefois que nul convive ne devienne le but d'une épigramme sanglante et envenimée. On n'entend là néanmoins ni orgues hydrauliques, ni concerts savants et étudiés. Pas de joueur de lyre, de joueur de flûte, de maître de chœur, de joueuse de sistre ou de tout autre instrument; le roi n'admet que les musiciens dont les sons ne plaisent pas moins à l'âme que les chants à l'oreille. »

13 Parmi ces amusements, les seuls qui ne sont pas exprimés par prétérition sont les mimici sales, ita ut nullus conviva mordacis linguae felle feriatur. De quoi s'agit-il? Selon les critiques, et notamment André Loyen ${ }^{10}$, il s'agirait de petits poèmes satiriques et plaisants, que le souverain tolère à condition qu'ils évitent de blesser. C'est sur cette question de l'illégalité ou du mordant de la satire que se cristallise l'un des points communs entre Théodoric et Majorien.

\section{Théodoric, un autre Majorien?}

On peut rapprocher cette attitude de celle de Majorien dans la lettre I, 11. Cette lettre est très longue, et s'achève par la description particulièrement mise en scène d'un banquet. Elle débute sur l'arrivée de Sidoine à Arles, où il est accueilli par une rumeur selon laquelle il aurait écrit une satire des plus venimeuses, dont il n'est cependant pas l'auteur. Cette accusation se déroule sur un fond d'intrigues et de cabales bien plus inquiétantes pour Sidoine, qui avait soutenu l'empereur détrôné par Majorien. Après la mise en place du décor est décrit un banquet, le soir même de l'arrivée de Sidoine, chez l'empereur Majorien, dont il a été panégyriste. Sidoine y compose une épigramme. Le contexte, s'il ressemble à celui du ludus propre à la création d'épigrammes, est plus grave. Sidoine est accusé par ses détracteurs d'avoir écrit une satire (satyrici uersus) particulièrement mordante, ce dont il n'est pas coupable. Mis en cause devant l'empereur et sa cour, Sidoine est sommé de s'expliquer. Pour ce faire, il écrit une épigramme qui à la fois discrédite ses adversaires et réfute les accusations de satire. 
L'accent semble être mis ici sur la liberté. Cependant, la permission absolue d'écrire (prohibiturum quin quae uelis scribas) est tempérée par le cum adversatif qui implique que la satire ne doit pas léser. Cette clémence n'est pas un vain mot puisque Sidoine se trouvait en fâcheuse posture, en tant que panégyriste de l'ancien empereur (Sidon., Ep. I, 11, 5) :

Et princeps, Deum testor et statum publicum, me de caetero nunquam prohibiturum quin quae uelis scribas, quippe cum tibi crimen impactum probari nullo modo possit ; simul et periniurium est sententiam purpurati tribuere priuatis hoc simultatibus, ut innocens ac secura nobilitas propter odia certa crimine incerto periclitetur.

«L'empereur: “Je prends Dieu et l'État à témoin, que jamais je ne t'empêcherai d'écrire ce que tu voudras, puisque l'on ne peut en aucune manière établir les accusations dirigées contre toi ; il serait aussi trop injuste que, laissant vivre des inimitiés privées, la noblesse innocente et tranquille se trouve confrontée à des haines certaines, sous prétexte d'un crime tout à fait incertain." ”

Théodoric est donc un personnage grandement mis en valeur, quoique barbare. On peut se demander si ce n'est pas là le reflet d'une sympathie particulière de Sidoine pour le personnage de Théodoric plus que pour les Wisigoths dans leur ensemble. En effet, les relations de Sidoine avec la royauté wisigothique se dégraderont singulièrement après l'assassinat de Théodoric II par son propre frère Euric. Celui-ci, après s'être emparé du pouvoir, entreprend la conquête d'un des derniers bastions romains de Gaule, Clermont-Ferrand, dont Sidoine est évêque. De surcroît, Euric est arien $^{11}$. Après la prise de Clermont, Sidoine est donc emprisonné et ne doit sa libération qu'à son entregent.

On constate que l'aspect hautement mélioratif de Théodoric en roi parfait n'est pas sans rappeler, sur certains points, des traits de caractère de l'empereur Majorien, mentionnés dans la lettre I, 11. Pour faire le panégyrique de ce roi, Sidoine n'use pas de la poésie, mais de la prose. Cependant, cette lettre, dans ses pratiques et sa structure, évoque les panégyriques impériaux. Dans l'Antiquité tardive, les lettres sont loin de constituer des objets privés, lieux d'une discussion qui se déroulerait uniquement entre un expéditeur et un destinataire. Elles sont toujours des reconstructions et des mises en scène a posteriori. La lettre I, 2 de Sidoine n'est pas si éloignée du panégyrique, puisque, selon certains critiques ${ }^{12}$, elle constitue un ouvrage de propagande en forme de mise en scène épistolaire. De surcroît, la structure de la lettre rappelle celle d'un panégyrique : introduction dédicatoire, suivie de la description physique de Théodoric, puis de ces " faits » sous la forme du récit de sa journée. Tout se passe comme si Sidoine ne pouvait décemment dédier une œuvre d'un grand genre à un roi barbare, et qu'il procédait à une miniaturisation, dans tous les sens du terme : miniaturisation de la forme, par la brièveté relative de la lettre, et miniaturisation du contenu et du genre (le genre épique devient épistolaire, le récit des hauts faits devient récit de la journée).

\section{Du panégyrique impérial à l'éloge royal}

Sidoine a écrit le panégyrique de trois empereurs, avec lesquels il entretenait des relations de diverses natures, plus ou moins proches et personnelles. Alexandre Renaud, dans son article sur la voix auctoriale dans les Panégyriques de Sidoine, décrit le panégyrique comme " un genre conventionnel, voire le genre conventionnel, l'un de ceux pour lesquels l'horizon d'attente est le plus fort ${ }^{13}$ ", à la fois du point de vue du contenu, du style et de la structure. Cependant, ainsi qu'il a souvent été mis en lumière 
notamment chez Sidoine Apollinaire, cela n'exclut pas une certaine dose d'originalité, apportée notamment par la présence de l'auteur et son jugement quant à l'objet du panégyrique. Or les panégyriques des trois empereurs Avitus, Majorien et Anthémius, s'ils entretiennent des points communs, ne proposent pas le même traitement de la figure triomphale de l'empereur. De surcroît, les différentes vertus présentées par Sidoine rappellent celles qu'il attribue à Théodoric II, faisant presque du roi arien un empereur chrétien et romain.

Dans ces trois panégyriques, Sidoine développe une série de qualités qui, mises bout à bout, pourraient conduire à la création d'un empereur parfait. Ces textes développent en effet chacun des qualités différentes, mais attribuables pour certaines à Théodoric. Quelles sont ces qualités? Peut-on considérer que Théodoric se rapproche plus d'un empereur ou d'un autre?

\section{Avitus et Théodoric : la ciuilitas domestique}

19 Le premier panégyrique dans l'ordre chronologique est celui d'Avitus ${ }^{14}$. Il se singularise par le fait qu'il est adressé non seulement à un empereur, mais encore à un parent, puisque l'empereur Avitus est le beau-père de Sidoine. Le panégyrique est donc adressé par le datif socero. La proximité avec le destinataire autorise Sidoine à louer les vertus domestiques du souverain. L'évocation de la chasse, qui fait d'Avitus une sorte de gentleman farmer, fait d'ailleurs écho à la référence à la chasse et au tir à l'arc dans le portrait de Théodoric. Il n'est pas décrit en priorité comme un guerrier, ainsi que le seront Anthémius et Majorien, mais surtout comme un citoyen modèle et un roi pacificateur. Une longue scène de ce panégyrique très vivant le représente en effet concluant la paix avec les Goths (Sidon., carm. 7, 460-468) :

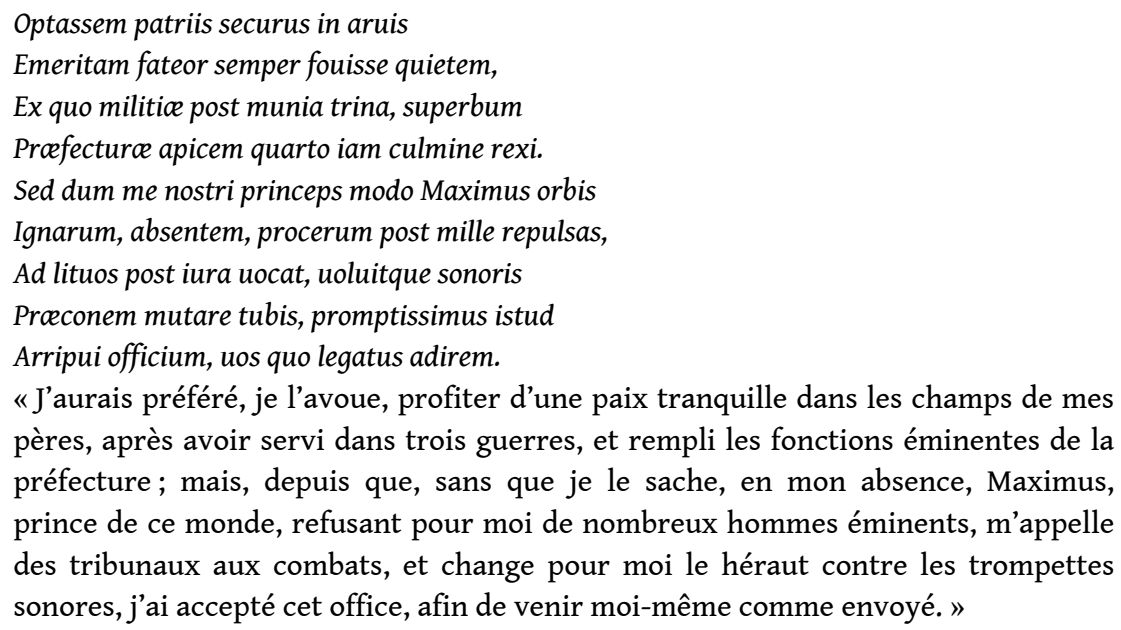

20 Le personnage qui permet la synthèse de ces deux aspects est celui de Cincinnatus. Consul et dictateur juste et efficace, c'est également un homme simple et modéré, comme Avitus et comme Théodoric. De plus, le panégyrique d'Avitus est l'occasion d'un éloge direct de Théodoric, lors de la description des pourparlers de paix avec Avitus. Le roi Théodoric II n'est pas nommé, mais dispose d'un portrait en creux par l'utilisation du discours direct. Théodoric y est présenté comme un roi lettré, mais aussi modéré et pacifique, sorte de double d'Avitus lui-même (Sidon., carm. 7, 496-500) :

paruumque ediscere iussit

Ad tua uerba pater, docili quo prisca Maronis

Carmine molliret Scythicos mihi pagina mores. 
Iam pacem tum uelle doces; sed percipe, qua sit

Conditio obsequii, forsan rata pacta probabis.

"Quand j'étais enfant, mon père m'ordonna d'apprendre près de toi les vers de Virgile, afin que ces pages anciennes adoucissent mes mœurs scythiques. Tu m'apprends que tu veux la paix maintenant. Mais écoute quelles sont les conditions de mon obéissance : peut-être les approuveras-tu.»

\section{Anthémius et Théodoric : des forces de la nature} apprentissage des belles-lettres, lors de son passage « du glaive à la pourpre » (Sidon., carm. 2, 182-192) :

Prceterea quidquid Latiaribus indere libris

Prisca cetas studuit, totum percurrere suetus :

Mantua quas acies, pelagique pericula lusit,

Smyrnceas imitata tubas : quamcunque loquendi

Arpinas dat consul opem; sine fine locutus

Fabro progenitus, spreto cui patre polita

Eloquiis plus lingua fuit ; uel quidquid in ceuum

Mittunt Euganeis Patauina uolumina chartis :

Qua Crispus breuitate placet, quo pondere Varro,

Quo genio Plautus, quo fulmine Quintilianus,

Qua pompa Tacitus, nunquam sine laude loquendus.

«Il étudiait encore tout ce que les âges anciens ont consigné dans les livres du Latium: les grands combats et les dangers des mers célébrés par le poète de Mantoue, imitant les trompettes de Smyrne; toutes les richesses d'éloquence que déroule le consul d'Arpinuna, ou cet orateur infatigable issu d'un forgeron, et qui, dédaignant l'art de son père, aima mieux s'exercer à polir le langage; tous les volumes enfin dont l'écrivain de Padoue a gratifié la postérité. Il méditait la brièveté de Salluste, le grave savoir de Varron, le génie de Plaute, l'éloquence foudroyante de Quintilien, la pompe de ce Tacite dont on ne doit jamais parler qu'avec éloge. »

De même, Théodoric, le monarque, montre à la fois une force physique et une force intellectuelle.

\section{Majorien et Théodoric, des alter ego?}

Le panégyrique de Majorien débute de façon beaucoup plus littéraire que les deux autres, par une préface imitée de Virgile, qui met d'emblée l'accent sur la production poétique. Le choix de la topique virgilienne n'est évidemment pas anodin, puisqu'il fait référence au début des Bucoliques et à la volonté de réconciliation nationale manifestée par Auguste, et donc par Majorien. La préface est construite en deux parties. La première offre le panégyrique à Pétrus, un ami de Sidoine. Il est comparé à Mécène, mais cette évocation renvoie bien sûr à Auguste, et par là à Majorien. La référence à la 
première Bucolique est également liée à l'idée de réconciliation nationale et de réorganisation de la libertas poétique mise en œuvre à l'avènement d'Auguste comme à l'avènement de Majorien (Sidon., carm. 3, 1-15) :

Quid faceret loetas segetes, quod tempus amandum

Messibus, et gregibus, uitibus, atque apibus,

Ad Meconatis quondam sunt edita nomen :

Hinc, Maro, post audes arma uirumque loqui.

At mihi Petrus erit Mecœnas temporis huius,

Nam famce pelagus sidere curro suo.

Si probat, emittit ; si damnat, carmina celat,

Nec nos ronchisono rhinocerote notat.

I, liber, hic nostrum tutatur, crede, pudorem.

Hoc censore etiam displicuisse placet.

Tityrus ut quondam patuloe sub tegmine fagi

Volueret inflatos murmura per calamos,

Prcestitit afflicto ius uitce Cosar et agri,

Nec stetit ad tenuem celsior ira reum :

Sed rus concessum dum largo in principe laudat,

Coelum pro terris rustica musa dedit.

Nec fuit inferius Phœbeia dona referre:

Fecerat hic dominum, fecit et ille deum :

Et tibi, Flacce, acies Bruti Cassiique secuto,

Carminis est auctor, qui fuit et uenice.

Sic mihi diuerso nuper sub Marte cadenti,

Iussisti placido, uictor, ut essem animo,

Seruiat ergo tibi seruati lingua potce,

Atque mece uitce laus tua sit pretium.

Non ego mordaci fodiam modo dente Maronem,

Nec ciuem carpam, terra Sabella, tuum.

Res minor ingenio nobis, sed Cosare maior;

Vincant eloquio, dummodo nos domino.

«Ce qui peut rendre les campagnes riantes, quel est le temps propice aux moissons et aux troupeaux, à la vigne et aux abeilles, Virgile autrefois enseignait tout cela, sous les auspices de Mécène, avant de parler des armes et du héros. Pétrus deviendra pour moi le Mécène de ce temps; il sera l'étoile qui me guidera sur l'océan de la renommée. S'il approuve un ouvrage, il le produit au grand jour ; s'il le condamne, il le tient caché, et ne l'accable pas du poids de sa critique. Va, mon livre ; Pétrus, n'en doute pas, encourage notre timidité, et, même, il me plairait de déplaire à un tel censeur. Lorsque autrefois Tityre, sous l'épais feuillage d'un hêtre, gonflait son chalumeau des sons les plus doux, César avait laissé à l'affligé la vie et le champ paternel ; la haute colère du prince n'avait pas tenu face aux supplications de l'humble proscrit. Mais, pendant que le pasteur loue le monarque libéral de lui avoir accordé son champ, la muse rustique donne le ciel au prix de la terre. Le don de Phœbus n'était pas inférieur. Celui-ci avait fait un maître, celui-là fit un dieu. Et toi, Flaccus, qui avais suivi les étendards de Brutus et de Cassius, le protecteur de ta muse fut celui qui œuvra à ton pardon. Ainsi, lorsque je succombais naguère sous des drapeaux opposés, tu m'ordonnas, ô vainqueur, de ne pas avoir peur. Qu'elle devienne donc ton esclave, la langue du poète que tu as sauvée, et que ton éloge soit le prix de ma vie. Je n'irai pas d'une dent maligne attaquer Virgile, ni ton poète, ô terre des Sabins. Si je n'ai pas leur talent, le César que je chante est plus grand que le leur; qu'ils l'emportent par l'éloquence, pourvu que nous l'emportions, nous, par la valeur de notre maître. »

La seconde partie de la préface insiste également sur la clémence de l'empereur. En effet, lorsqu'il écrit le Panégyrique de Majorien, Sidoine se trouve dans une situation difficile : il a soutenu très étroitement l'empereur précédent, et doit donc se montrer 
particulièrement appliqué dans l'éloge de son successeur afin de lui prouver son allégeance.

Le traitement du personnage de Théodoric, roi des Wisigoths, par Sidoine Apollinaire est révélateur à bien des égards. Tout d'abord, il témoigne d'un changement de mentalité à l'égard des barbares de la part d'un aristocrate comme Sidoine, à une époque où disparaît le pouvoir impérial et où règnent des rois barbares. On peut cependant se demander dans quelle mesure l'attitude de Sidoine vis-à-vis du roi Théodoric ne relève pas également en partie d'une accointance personnelle avec ce roi barbare, puisque Sidoine n'a pas eu le même discours concernant le personnage d'Euric, frère et successeur de Théodoric (avec lequel il a entretenu des relations bien moins harmonieuses, puisque celui-ci l'a fait emprisonner après la prise de ClermontFerrand).

De plus, il interroge le mélange des genres dans l'Antiquité tardive. En cette période où la hiérarchie des genres est bouleversée, le panégyrique, grand genre par excellence, par sa forme et son sujet, se trouve parasité par l'épistolaire, qui se met chez Sidoine à assumer le rôle encomiastique, alors que les panégyriques se font beaucoup plus nuancés et ironiques. Il est également possible que Sidoine n'ait pas osé traiter par un panégyrique, genre impérial par excellence, le portrait d'un roi malgré tout barbare.

\section{BIBLIOGRAPHIE}

\section{Textes anciens}

C. Solius Apollinaris Sidonius, recensuit P. MOHR, Leipzig, Teubner, 1895.

Sidonius, Poems and Letters, with an english transl., introd. and notes by W. B. ANDERSON, The Loeb Classical Library, Londres, W. Heinemann, 1936-1965, 2 vol.

Sidoine Apollinaire, texte établi et trad. par A. LOYEN, CUF, Paris, 1960-1970, 3 vol.

\section{Études modernes}

BANNIARD M. 1992, « La rouille et la lime : Sidoine Apollinaire et la langue classique en Gaule au ve siècle ", in L. HOLTZ, J.-C. FREDOUILLE (éd.), De Tertullien aux Mozarabes : Antiquité tardive et christianisme ancien. Mélanges offerts à Jacques Fontaine à l'occasion de son $70^{e}$ anniversaire par ses élèves, amis et collègues, I, Coll. des Études augustiniennes, Paris, Institut des Études augustiniennes, p. 413-427. 
BONJOUR M. 1982, « Personnification, allégorie et prosopopée dans les Panégyriques de Sidoine Apollinaire ", Vichiana 11, p. 5-17.

GIBBON E. 1993, The History of the Decline and Fall of the Roman Empire, H. Trevor Roper (éd.), Everyman's Library 115, New York, A. A. Knopf.

GOSSEREZ L. 2009, « Mythe et politique dans le panégyrique d'Avitus », VL 180, p. 39-52.

KAUFMANN F.-M. 1995, Studien zu Sidonius Apollinaris, Europäische Hochschulschriften. Reihe 3, Geschichte und ihre Hilfswissenschaften, Francfort, P. Lang.

LOYEN A. 1943, Sidoine Apollinaire et l'esprit précieux en Gaule aux derniers jours de l'Empire, Coll. d'études latines, Paris, Les Belles Lettres.

RENAUD A. 2009, « La voix du poète dans les Panégyriques de Sidoine Apollinaire », VL 180, p. 53-63.

REYDELLET M. 1981, La royauté dans la littérature latine, de Sidoine Apollinaire à Isidore de Séville, Bibliothèques des Écoles françaises d'Athènes et de Rome 243, Rome, École française de Rome. STEVENS C. E. 1933, Sidonius Apollinaris and His Age, Oxford, Clarendon Press.

\section{NOTES}

1. Pour le texte des lettres et des Panégyriques on a utilisé l'édition Teubner 1886.

2. REYDELLET 1981, p. 49.

3. On peut se reporter par ex. à la description d'Hannibal chez Tite-Live (Liv., 21, 4).

4. On peut penser au portrait d'Arminius chez Tacite (Tac., Ann. 1, 55).

5. Voir également REYDELLET 1981, p. 69.

6. GIBBON 1993, p. 1111. On peut également consulter à ce sujet l'ouvrage de KAUFMANN 1995.

7. REYDELLET 1981, p. 71, et STEVENS 1933, p. 24.

8. Quint., Inst. 2, 15, 35.

9. Voir aussi BANNIARD 1992.

10. Voir LOYEN 1943.

11. L'arianisme est un courant de pensée des débuts du christianisme, dû à Arius, théologien alexandrin au début $d u \mathrm{IV}^{\mathrm{e}} \mathrm{s}$., et dont le point central concerne les positions respectives des concepts de "Dieu le père " et de "son fils Jésus». La pensée de l'arianisme affirme que, si Dieu est divin, son Fils, lui, est d'abord humain, même s'il possède une part de divinité.

12. REYDELLET 1981, p. 72.

13. RENAUD 2009, p. 53.

14. Sur le panégyrique d'Avitus, voir BONJOUR 1982 et GOSSEREZ 2009. 


\section{RÉSUMÉS}

Dans l'imaginaire populaire, le Romain voit le Barbare comme un être dépourvu de raffinement. Sidoine Apollinaire quant à lui n'était peut-être pas de cet avis. Le portrait qu'il dresse du personnage de Théodoric, roi des Wisigoths, dans ses lettres I, 2 et I, 11 ainsi que dans ses panégyriques impériaux, est de fait révélateur à bien des égards. Il témoigne en premier lieu d'un changement de mentalité envers les barbares de la part d'un aristocrate comme Sidoine, à une époque où disparaît le pouvoir impérial et où règnent des rois barbares. De plus, ce traitement de l'image des barbares interroge le mélange des genres dans l'Antiquité tardive. En cette période où la hiérarchie des genres est bouleversée, le panégyrique, grand genre par excellence, par sa forme et son sujet, se trouve parasité par l'épistolaire, qui se met chez Sidoine à assumer le rôle encomiastique, alors que les Panégyriques se font beaucoup nuancés et ironiques.

In our collective mind, Romans were supposed to consider the Barbarians as unrefined being. Sidonius Apollinaris may not have shared this point of view. The portrait he draws of Theodoric, king of the Visigoths (in his letters 1,2, and 1,11 as in his imperial panegyrics), is indeed very instructive. It shows a change in the attitude of Romans towards Barbarians, in a time when imperial power disappears, replaced by Barbarian kings. Besides, it questions the concept of mixture of literary styles during Late Antiquity. In this period, the epistolary genre interferes with the panegyric, great genre par excellence, the first handling the encomiastic role, as the Panegyrics become more ironic and tempered.

\section{INDEX}

Index géographique : Arles, Clermont-Ferrand nomsmotscles Ammien Marcellin, Aristote, Plaute, Quintilien, Sidoine Apollinaire, Salluste, Suétone, Tacite, Tite-Live, Varron

Keywords : Panegyric, epistolary genre, Theodoric

Mots-clés : Panégyrique, genre épistolaire, Théodoric

\section{AUTEURS}

\section{CAMILLE BONNAN-GARÇON}

Université Jean Moulin Lyon 3 